\title{
Rayleigh-Wave Multipathing along the West Coast of North America
}

\author{
by Chen Ji, Seiji Tsuboi, Dimitri Komatitsch, and Jeroen Tromp
}

\begin{abstract}
We have created a movie of surface ground motion for the 3 November 2002 Denali fault earthquake based on spectral-element simulations using crustal model CRUST2.0, mantle model S20RTS, topography and bathymetry model ETOPO5, and a finite-fault slip model. The movie features two anomalous wave packets that travel along the west coast of the North American plate following offgreat-circle paths. These wave packets are Rayleigh waves with dominant periods around $20 \mathrm{sec}$, which are also found in seismograms recorded by the Southern California Seismic Network. One of these packets is the direct surface wave, whose group arrival time changes laterally as dictated by the shape of the Oregon coast. The other packet is a surface wave reflected by a lateral interface underneath the Rocky Mountains. A linear reflector parallel to the Canadian coast offsetting it by a few hundred kilometers can explain its arrival time, but the offsetting distance derived from the synthetic seismograms puts the reflector $350 \mathrm{~km}$ northeast of the result obtained from the data, indicating a need to update the crustal and mantle models in this area.
\end{abstract}

Online material: Animation of normalized simulated vertical displacement for the 2002 Denali earthquake.

\section{Introduction}

It has long been recognized that lateral velocity gradients in the lithosphere can refract and reflect intermediate surface waves (Evernden, 1953; Knopoff et al., 1966), although such effects are not as well documented as for body waves (Nakanishi, 1992). Evidence has been presented suggesting that refraction and reflection may occur at various tectonic boundaries, such as continental margins (Capon, 1970; Levshin and Berteussen, 1979), ridges (Capon, 1970), and sea trenches. Numerous theoretical studies have been conducted to explain surface-wave amplitude variations, phase delays, and arrival-azimuth anomalies (Snieder, 1986; Woodhouse and Wong, 1986; Laske, 1995).

In general, crustal structures beneath mountain ranges are distinctly different from surrounding regions because of the presence of a mountain root and the uplift of basement rock. Tall mountain ranges should therefore be associated with strong lateral phase-velocity variations for intermediate surface waves. To our knowledge, surface-wave multipathing due to crustal heterogeneities associated with mountains has thus far not been reported in the literature.

In this article, we examine short-period Rayleigh waves propagating along the western boundary of the North American plate. We show that multipathing effects of 20-sec Rayleigh waves are observed in a movie of simulated ground motion as well as in Southern California Seismic Network (SCSN) data for the M 7.93 November 2002 Denali fault, Alaska, earthquake. Using data from this large aperture seis- mic network, we are able to determine the location of a possible reflector.

\section{Numerical Simulations of Ground Motion}

We have calculated the synthetic seismic wave field for the 3 November 2002 Denali fault earthquake using the spectral-element method (SEM) (Komatitsch and Tromp, 2002) on the Japanese Earth Simulator (Tsuboi et al., 2003), which was in 2004 the fastest computer in the world. The SEM is a high-degree version of the finite-element method that has the useful property of yielding an exactly diagonal mass matrix. Our simulations take into account 3D mantle model S20RTS (Ritsema et al., 1999), crustal model CRUST2.0 (Bassin et al., mahi.ucsd.edu/Gabi/rem.html, 2001), topography and bathymetry model ETOPO5, and a kinematic slip model of the earthquake (Ji et al., 2004). Based on these simulations we can accurately model broadband teleseismic body waves $(f<0.2 \mathrm{~Hz})$ and long-period surface waves ( $T>40 \mathrm{sec}$ ) (Tsuboi et al., 2003). (E) A movie showing the vertical component of displacement is available in the electronic edition of BSSA.)

Figure 1 shows a snapshot of the vertical displacement 20 min after the initiation of the earthquake, when the direct $P$ and $S$ waves have already traveled across the North American continent. Figure 1 is therefore dominated by the motion of Rayleigh waves. In particular, the high-amplitude and 


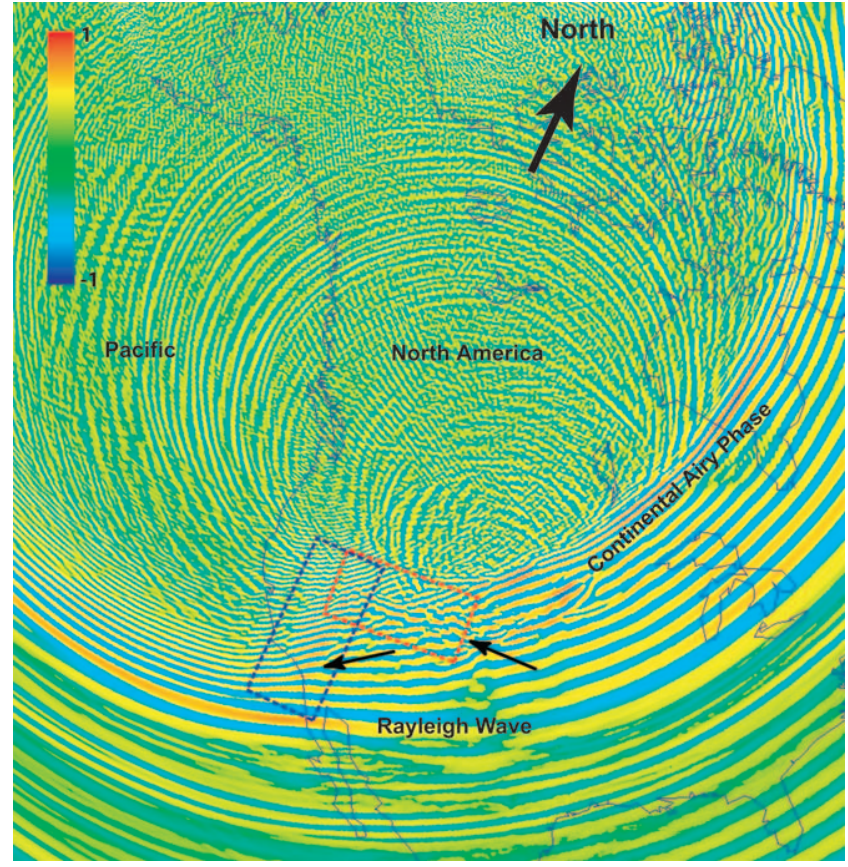

Figure 1. Snapshot of a numerical simulation of ground motion for the 3 November 2002 Denali fault earthquake $20 \mathrm{~min}$ after the origin time. The North American coastline is delineated in blue. The colors show normalized vertical displacement. The direct long-period Rayleigh wave and continental Airy phase are labeled. The two dashed boxes highlight anomalous wave packets referred to in the text as the Oregon packet (blue box) and the Rocky Mountain packet (red box). The two small black arrows indicate the orientations of the wave fronts of these anomalous wave packets.

long-wavelength wave packet near the Gulf of California is the long-period fundamental Rayleigh wave ( $T>40 \mathrm{sec})$. Another high-amplitude but short-wavelength wave packet is the so-called continental Airy phase (e.g., Lay and Wallace, 1995). The shape of their respective wavefronts indicates that these waves propagate roughly along the greatcircle path from the epicenter. However, we also observe two anomalous wave packets (dashed boxes, Fig. 1) that propagate off the great circle. The blue box highlights a wave packet whose phase trends along a line roughly parallel to the Oregon coast (hereafter referred to as the "Oregon" packet). The red box shows another wave packet that travels southward (hereafter referred to as the "Rocky Mountain" packet). We were curious about their origin, which motivated us to search for similar waves in broadband waveforms recorded by the dense SCSN (Fig. 2) (Hauksson et al., 2001).

A snapshot of the wave field illustrates the spatial distribution of ground motion at a given time, whereas a seismogram displays the temporal variation of ground motion at a given location. For surface waves we can convert distances between wave packets into temporal intervals in seismograms by dividing the former by a properly chosen phase

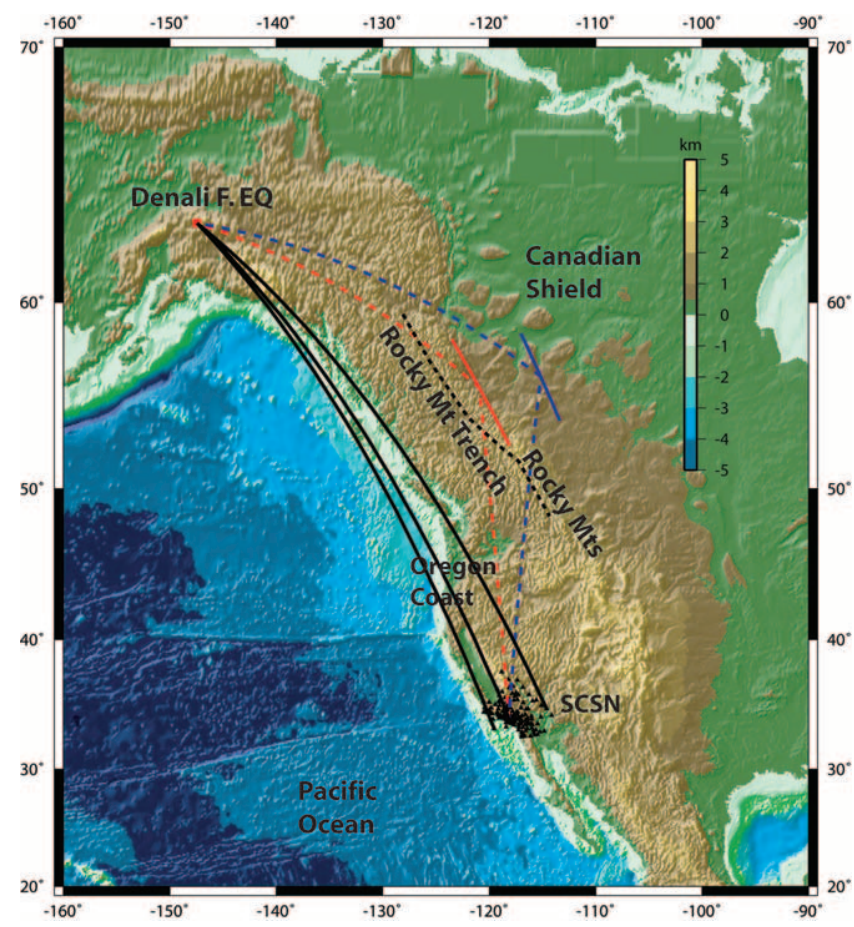

Figure 2. Stations of the Southern California Seismic Network (SCSN; filled triangles) and epicenter of the 3 November 2002 Denali fault, Alaska, earthquake (Denali F. EQ; red square) superimposed on the ETOPO5 topography and bathymetry map using a Mercator projection. Black lines show representative great-circle paths. The blue and red bars show the reflectors inferred from the synthetic seismograms and the data, respectively. Dashed lines indicate the reflected ray paths for one representative station, PAS (Pasadena, California). Several geographic features such as the Oregon Coast, the Rocky Mountains (Rocky Mts), and the Rocky Mountain Trench (Rocky Mt Trench; black dashed line) are also indicated.

or group velocity. For instance, in Figure 1 the Oregon packet is closer to the epicenter than the long-period Rayleigh waves, and their relative distance gradually increases when moving from the coastline further inland. If one wants to look for such a pattern in the seismograms recorded at stations of the SCSN, the Oregon packet should arrive later than the long-period Rayleigh wave, and the temporal spacing should increase when one moves from stations located near the coast to stations located further inland. An opposite trend should be associated with the Rocky Mountain wave packet.

We can measure wavelengths and wavefront orientations of surface waves based on snapshots. In Figure 1, the dominant wavelength of a wave packet may be determined by counting cycles and measuring distances. We determined that the dominant wavelength of the Oregon and Rocky Mountain packets is about one third of that of the longperiod Rayleigh wave, close to that of the continental Airy phase (Fig. 1). Therefore, the dominant period of the anom- 
alous wave packets should be much shorter than that of the long-period Rayleigh waves. The standard definition of the wavefront is in terms of the loci of points that undergo the same motion at a given time (e.g., Lay and Wallace, 1995). In a snapshot, this corresponds to the loci of the points with the same color. We find that the wavefront of the Oregon packet is rotated counterclockwise relative to that of the long-period (fundamental) Rayleigh wave. In contrast, the wavefront of the Rocky Mountain packet is rotated clockwise by $30^{\circ}$ relative to the long-period Rayleigh wave front. Keeping these relationships in mind, we can now analyze the seismograms recorded by the SCSN.

\section{Analysis of SCSN Data}

We downloaded broadband waveforms from the Southern California Earthquake Data Center (SCEDC) and converted them to surface displacements by removing the instrument response. The resulting displacements were subsequently compared with synthetic seismograms at the same stations calculated in a previous study (Tsuboi et al., 2003).

The red square in the left panel of Figure 2 indicates the epicenter of the 3 November 2002, Denali fault, Alaska, earthquake. The black lines represent typical great-circle paths. Although this event involves a $320-\mathrm{km}-$ long fault plane consisting of several segments (Eberhart-Phillips et al., 2003), it is reasonable to ignore its finiteness in our study because of the large epicentral distances considered $(\sim 4000 \mathrm{~km})$ and because of the small variations in source azimuth $\left(<8^{\circ}\right)$. The great-circle paths of most SCSN stations for this earthquake involve both the North American continent and the Pacific ocean. The ratio of the two parts varies considerably with azimuth because of the shape of the Oregon coast.

We find that it is not easy to identify anomalous signals directly from the broadband data (Fig. 3). However, after using a narrow bandpass filter with corner frequencies of $0.04 \mathrm{~Hz}$ and $0.06 \mathrm{~Hz}$, these signals become the dominant phases in the seismograms (Fig. 4). We subsequently calculate envelope functions of the bandpassed signals to highlight their group motion (e.g., Lay and Wallace, 1995).

The envelope profiles of the vertical component seismograms are shown in the middle and right panels of Figure 5. To investigate the temporal separation between the long-period Rayleigh waves and the anomalous wave packets, we align the data and the synthetic seismograms using a reduced velocity of $3.8 \mathrm{~km} / \mathrm{sec}$, which is roughly the average group velocity of long-period Rayleigh waves in this region, as illustrated in Figure 3. We sort the seismograms based on the length of the continental portion $\left(D_{\mathrm{c}}\right)$ of the entire great-circle path $(D)$, which is approximated here as the part of the great-circle path with positive ground eleva-
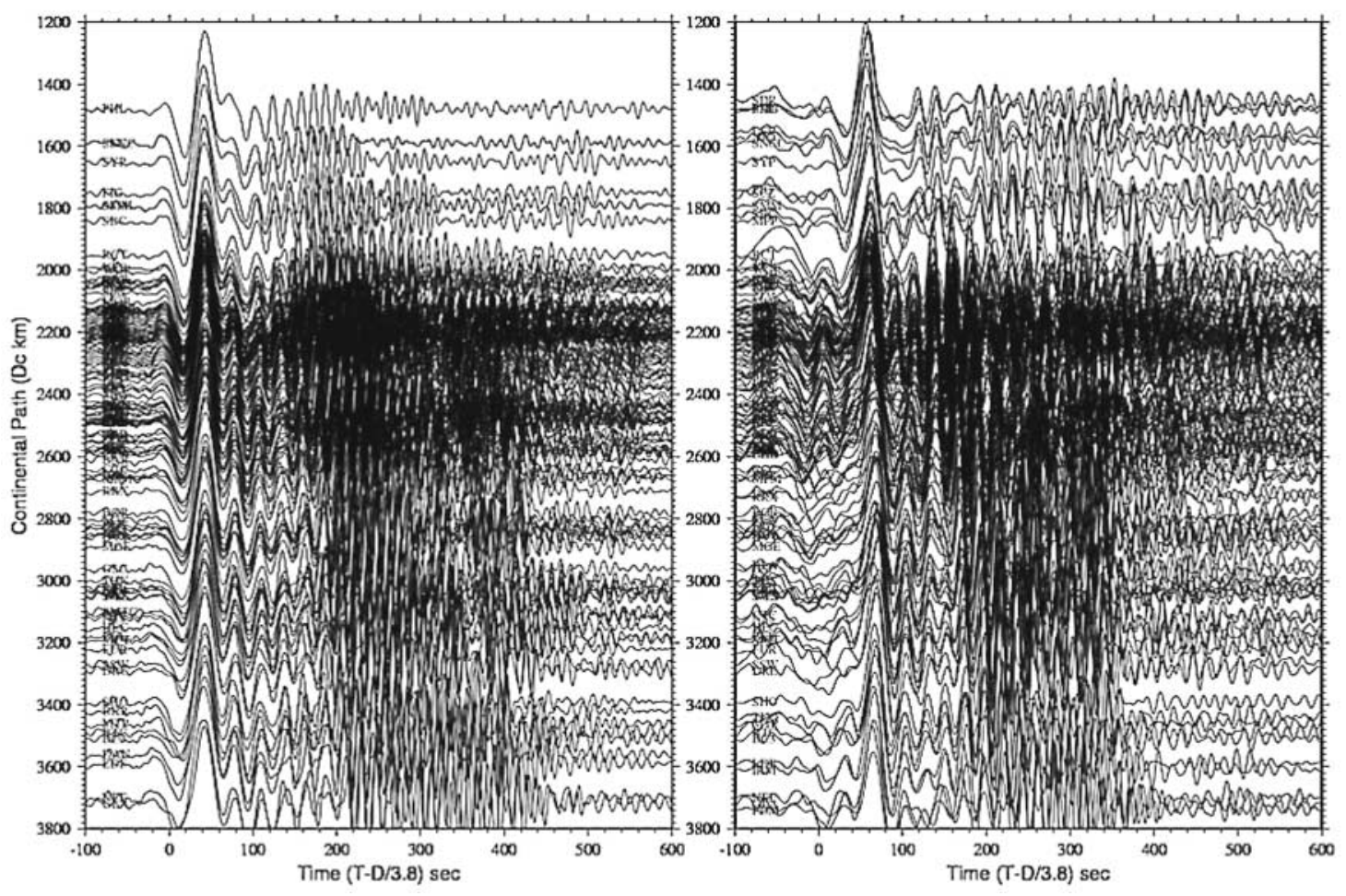

Figure 3. Vertical displacement SEM synthetic seismograms (left) and SCSN data (right) for the 3 November 2002, Denali fault, Alaska, earthquake. Both synthetic seismograms and data have been lowpass filtered with a corner period of $10 \mathrm{sec}$. The seismograms are sorted based on the continental portion $D_{c}$ of their great-circle path and aligned based on a reduced velocity of $3.8 \mathrm{~km} / \mathrm{sec}$. 


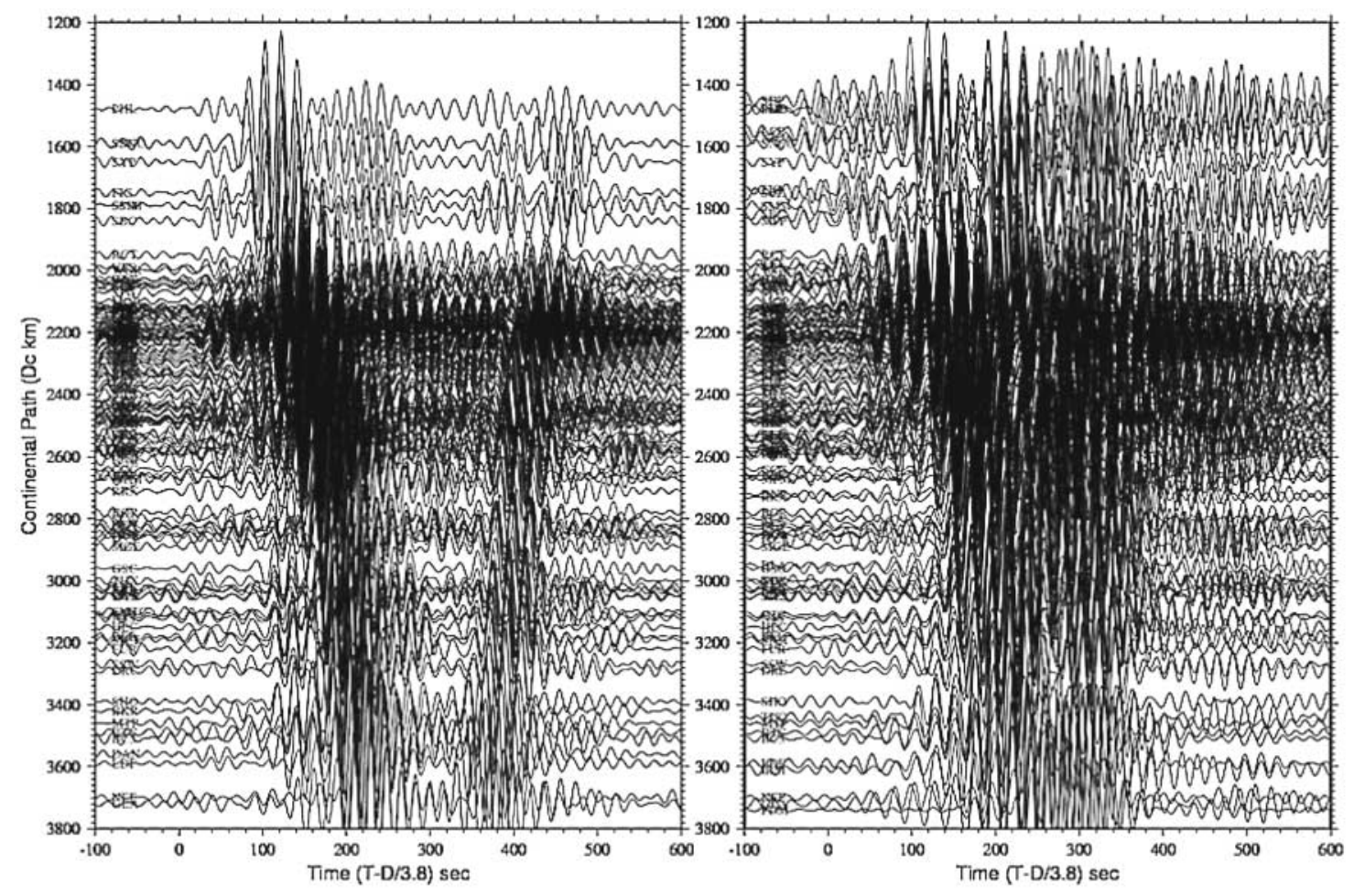

Figure 4. Bandpass-filtered vertical displacement SEM synthetic seismograms (left) and SCSN data (right) for the 3 November 2002 Denali fault, Alaska, earthquake. Both synthetic seismograms and data have been bandpass-filtered between $0.04 \mathrm{~Hz}$ and 0.06 $\mathrm{Hz}$. The seismograms are sorted based on the continental portion $D_{c}$ of their greatcircle path and aligned based on a reduced velocity of $3.8 \mathrm{~km} / \mathrm{sec}$.

tion. Considering the shape of the Oregon coast and the small spatial extent of the SCSN (Fig. 2), stations with a larger $D_{\mathrm{c}}$ are generally located further away from the California coast. Therefore, the Oregon wave packet in Figure 5 should arrive later when $D_{\text {c }}$ increases, while the Rocky Mountain wave packet should arrive earlier under the same circumstances. The synthetic seismograms show two distinct wave packets whose characteristics are what we expect from the movie and the snapshot in Figure 1.

\section{The Oregon Packet}

We first attempt to fit the trend of the Oregon packet based on a simple function $t_{b}$, indicated by the solid red lines in Figure 5, as

$$
t_{b}\left(D_{c}, D_{o}\right)=D_{c} / V_{c}+D_{o} / V_{o} .
$$

Here $D_{\mathrm{o}}$ denotes the length of the oceanic part of a greatcircle path and $V_{c}$ and $V_{o}$ are the Rayleigh-wave group velocities for the continental and oceanic parts of the path, respectively. The trend of the Oregon packet is well fit using $3.1 \mathrm{~km} / \mathrm{sec}$ for $V_{c}$ and $3.7 \mathrm{~km} / \mathrm{sec}$ for $V_{o}$ (Fig. 5). Both values are slightly higher than typical continental/oceanic Rayleigh-wave group velocities at $20 \mathrm{sec}$ (Oliver, 1962) due to the slow mantle velocities in this region [Ritsema et al.,
1999]. The quality of the fits is further evaluated in Figure 4 , where we align envelope functions of both recorded data and synthetic seismograms using equation (1) and sort them as a function of source azimuth. We find that the fits to the data become worse when the azimuth is larger than $130^{\circ}$ or smaller than $135^{\circ}$, where the great-circle paths are close to the California or Canadian coast, respectively. The difference between the synthetic seismograms and the data suggests that crustal model CRUST2.0 needs to be refined in these regions.

The fits to the arrival time suggest that the Oregon wave packet represents the direct Rayleigh wave whose ray path is distorted by the ocean-to-continent transition. Because the group velocity-dispersion relationship for Rayleigh waves has a local minimum at about $20 \mathrm{sec}$ in the case of a continental path, significant energy arrives at a specific time, producing an amplification and interference effect called the Airy phase, as mentioned above. This is clearly visible in the movie of the numerical simulation (E) available in the electronic edition of BSSA), which shows that the Oregon packet gets generated as the Rayleigh wave crosses the Oregon coast.

Next, we determine the polarization of the synthetic and recorded wave packets. Because of the radiation pattern, the Love wave dominates the horizontal components and its coda overlaps with the Oregon Rayleigh-wave packet. Be- 

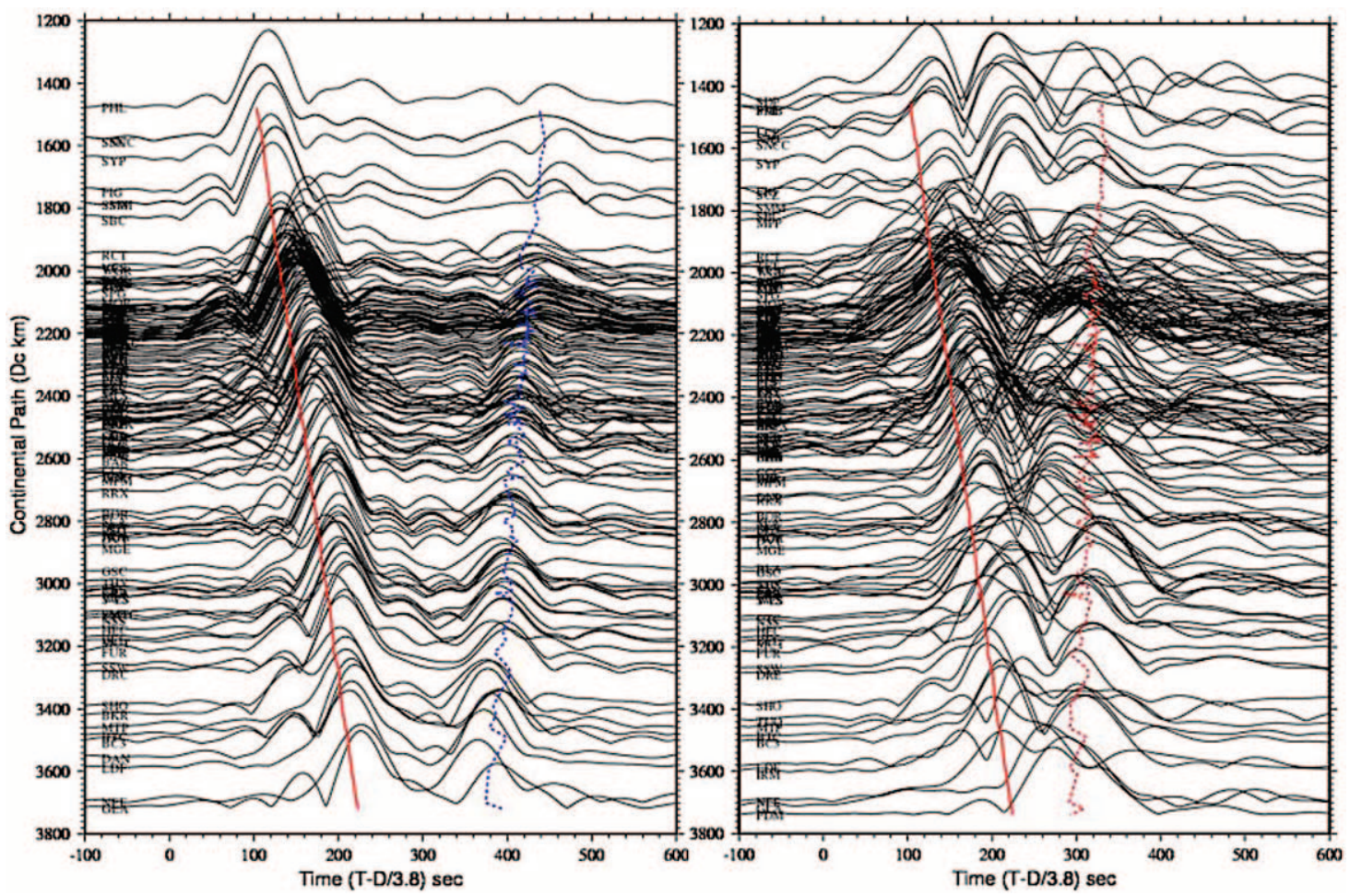

Figure 5. Envelopes of bandpass-filtered vertical displacement SEM synthetic seismograms (left) and SCSN data (right) for the 3 November 2002, Denali fault, Alaska, earthquake. The seismograms are sorted based upon the continental portion $D_{c}$ of their great-circle path and aligned based on a reduced velocity of $3.8 \mathrm{~km} / \mathrm{sec}$. The red lines indicate the predicted time of the "Oregon" wave packet computed based on equation (1). The dashed curves show the predicted arrival times of reflected waves computed based on equation (2) and a group velocity of $3.1 \mathrm{~km} / \mathrm{sec}$.

cause of heterogeneity, the Love-wave coda and the Rayleigh wave do not necessarily have perfectly perpendicular displacements in the horizontal plane. Various polarization analysis techniques based upon three-component data from a single station proved to be unstable (Cotte et al., 2000). We therefore decided to determine the phase-velocity vector within the neighborhood of a given station by using a method similar to what was proposed by Cotte et al. (2000) (see the Appendix for details). In Figure 3, colors are used to represent the off-great-circle arrival angles. The value is positive if the deviation near a station is clockwise relative to its great-circle path. Analysis of the synthetic seismograms (Fig. 6, left panel) shows counterclockwise off-greatcircle polarization angles associated with the Oregon packet at most stations, decreasing with source azimuth from $-5^{\circ}$ to nearly $-25^{\circ}$, consistent with the pattern shown in the snapshot (Fig. 1). For a given station, the off-great-circle angle of the Oregon packet is not constant but rather increases with time (Fig. 6). Analysis of recorded data (Fig. 6, right panel) shows a similar pattern in the middle portion of the profile (source azimuths ranging from $131.5^{\circ}$ to $135^{\circ}$ ). However, very large discrepancies occur outside this region, coincident with the discrepancies in the shape and arrival time of the envelope functions (Fig. 6). We will address these discrepancies after the discussion of the Rocky Mountain wave packet.

Large counterclockwise deviations from the great-circle path have been previously noticed at SCSN station PAS (Pasadena, California) for intermediate-period Rayleigh waves generated by Alaskan earthquakes (Zhang et al., 2003). They interpreted this observation as a result of the ocean-continent transition, which is verified by our $3 \mathrm{D}$ simulation (Figs. 1 and 6). The variation of off-great-circle angles with azimuth shown in Figure 6 is likely a result of wavefront healing. The variation with time at some stations probably indicates multipathing.

\section{The Rocky Mountain Packet}

The Rocky Mountain wave packet is also present in the recorded seismograms, but its temporal separation from the Oregon packet is much smaller than in the synthetic seismograms (Figs. 5 and 6). Coincidently, the polarization analysis of the synthetic seismograms shows large clockwise offgreat-circle deviations at almost all stations $\left(25-40^{\circ}\right)$, but the analysis of recorded data suggests that the Rocky Mountain packet propagates along a path just slightly off the great circle. 


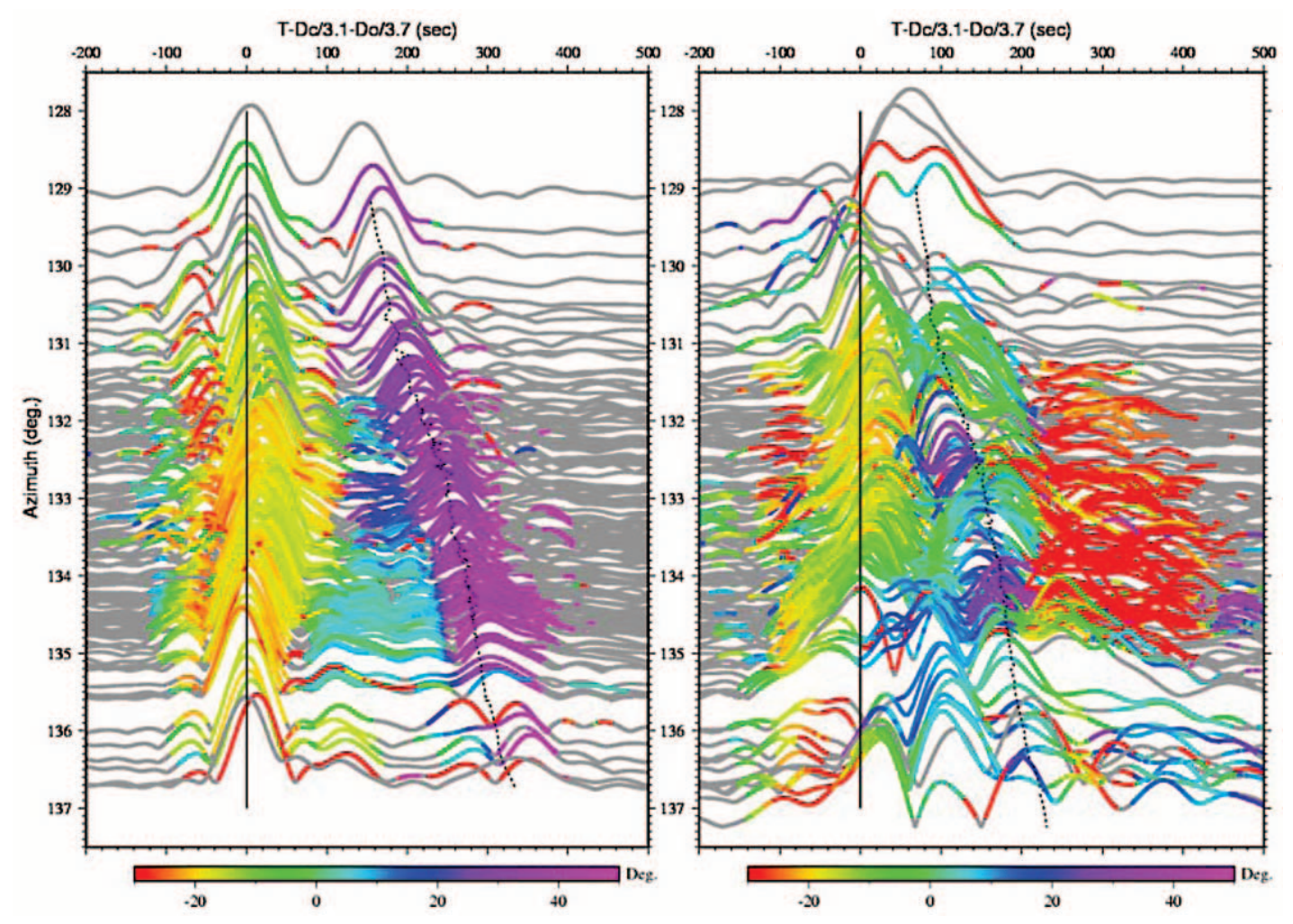

Figure 6. Source azimuthal profiles of the envelope function for the SEM synthetic displacement seismograms (left) and recorded data (right). Time is reduced based on equation (1). The colors show the off-great-circle arrival angles. Gray colors are used when the station coverage at this location did not provide a reliable estimate or if the amplitude of the envelope function at that instant time is less than $10 \%$ of its peak value. The dashed lines show the predicted arrival times of the "Rocky Mountain" reflected phases computed based on equation (2) and a group velocity of $3.1 \mathrm{~km} / \mathrm{sec}$.

Because the backazimuths of the SCSN stations vary from $332^{\circ}$ to $338^{\circ}$ with an average of $335^{\circ}$, the results of the polarization and snapshot analyses (Fig. 1) suggest that the Rocky Mountain wave packet originates to the north of the SCSN. We can therefore attempt to locate the associated reflector based on the arrival time of the Rocky Mountain packet (Figs. 5 and 6). We assume that the reflector is parallel to one particular reference great-circle path with a source azimuth of $\Theta_{0}$, but offset to the east by a distance of $H$ (see the cartoon in Fig. 7). Taking advantage of the small azimuthal variations $\left(<8^{\circ}\right)$ across the SCSN, we can ignore spherical effects and approximate the arrival time of the Rocky Mountain packet at a given station based on the following Cartesian relationships:

$$
\begin{aligned}
\xi & =\theta-\theta_{0} \\
t_{r} & =\sqrt{(L \cos \xi)^{2}+(2 H+L \sin \xi)^{2}} / V_{c}
\end{aligned}
$$

where $\theta$ denotes the azimuth, $L$ is the length of the greatcircle path, $t_{r}$ is the arrival time of the reflected phase using the group velocity $V_{c}$. By fitting the peak time of the synthetic Rocky Mountain packet in Figures 5 and 6, we find that the optimal value of $\theta_{0}$ is $129^{\circ}$, and that of $H$ is $950 \mathrm{~km}$, using $3.1 \mathrm{~km} / \mathrm{sec}$ as the group velocity. The dashed lines in Figures 5 (middle panel, blue dashed line) and 6 (left panel, black dashed line) are the predicted arrival times of the envelope peaks, which fit the synthetic profile very well. We further determine the locations of the reflection point for each SCSN station, which delineate a 600-km-long segment represented by the blue bar in Figures 2 and 7 (top).

If the Rocky Mountain packet in the recorded seismograms is similarly caused by a reflector, we should be able to determine its location as well. The optimal value of $H$ we find is only $600 \mathrm{~km}$ based on the same value of $3.1 \mathrm{~km} / \mathrm{sec}$ for the group velocity, i.e., smaller (by $350 \mathrm{~km}$ ) than the result estimated based on the synthetic seismograms, which was $950 \mathrm{~km}$. The predicted arrival time of the envelope peaks is plotted in Figures 5 (right panel, red dashed line) and 6 (right panel, black dashed line) and fits the recorded data, but not as well as in the synthetic seismograms. Note that the value of $H$ depends on the choice of the average group velocity, but that the difference between the results based on the synthetic seismograms and recorded data is on the contrary very robust. If we use a slower group velocity of $3.0 \mathrm{~km} / \mathrm{sec}$, the optimal $H$ predicted based on the synthetic seismograms changes to $800 \mathrm{~km}$, and that predicted based 


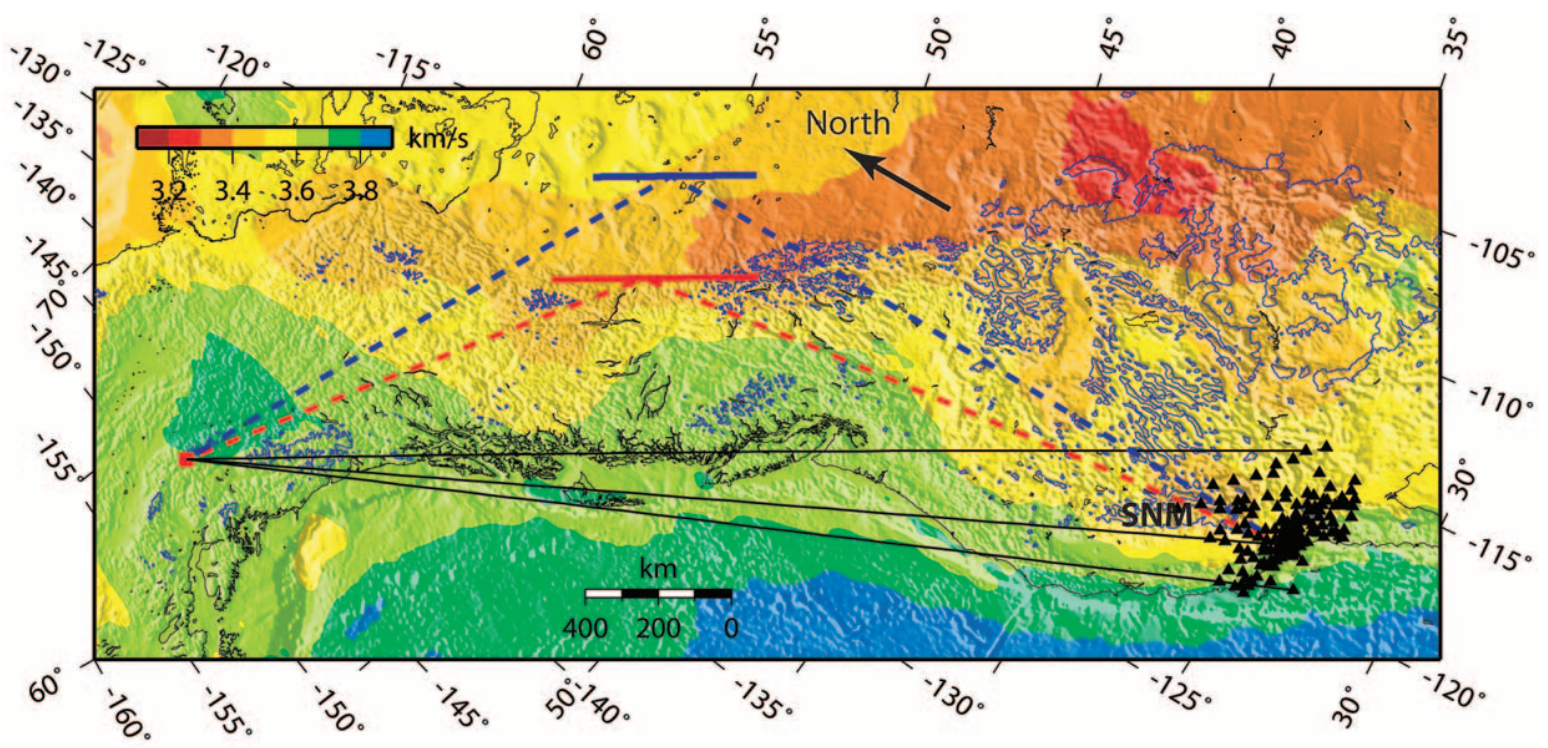

\section{Reflector}

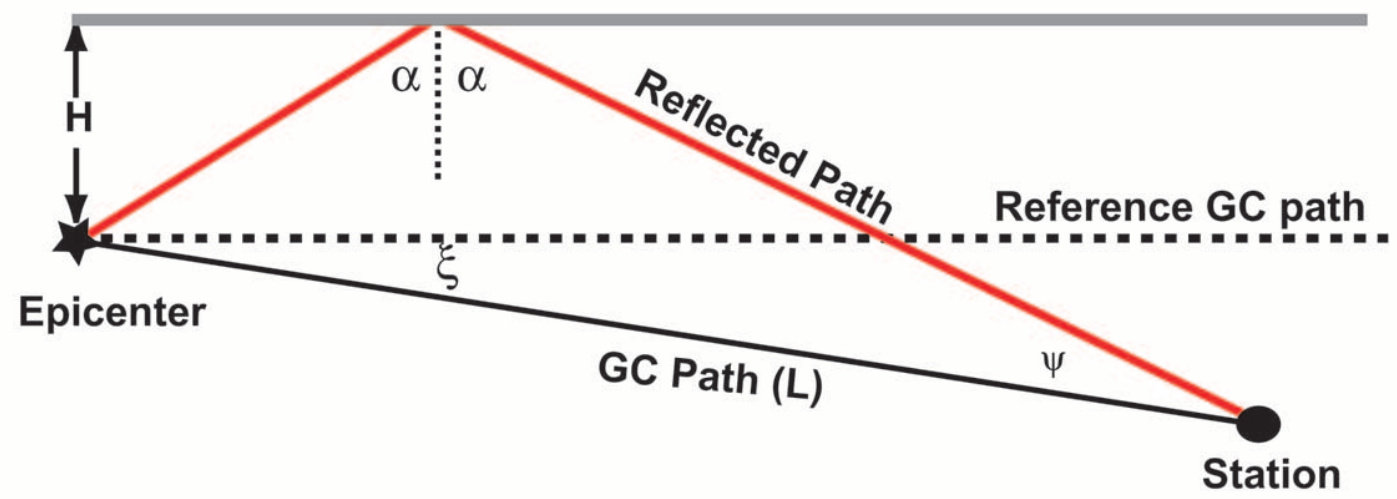

Figure 7. Upper panel: phase velocity of fundamental Rayleigh waves at $20 \mathrm{sec}$ calculated based upon the same 3D velocity model as used in the SEM simulation. The black triangles indicate the locations of the SCSN stations. The black lines are greatcircle paths, which are straight lines in this Oblique Mercator projection. The blue and red bars indicate the position of the reflector inferred from the analysis of the synthetic and observed profiles, respectively. The blue contours indicate mountains higher than $2000 \mathrm{~m}$. The location of the Sierra Nevada Mountains (SNM) is also labeled. Lower panel: geometric approach to calculating the length of a reflected path (red line). The dotted line shows the reference great-circle (GC) path, which is parallel to the reflector (thick gray line) and offsets by a distance $H$. The length of the great-circle path for an arbitrary station is denoted by $L$, and $\xi$ is the angle between this great-circle path and the reference path (see the main text for further details).

on the data drops to $440 \mathrm{~km}$, but the difference is $360 \mathrm{~km}$, i.e., it differs by only $10 \mathrm{~km}$ from the first result.

Alternatively, one can use the travel time of the reflected wave packet to a given station to define an ellipse on which the scatterer responsible for the arrival must be located. The envelope of the scattering ellipses for all the stations delineates the reflector. This procedure is illustrated in Figure 8, in which the scattering ellipses are denoted in grey. We used a group velocity of $3.1 \mathrm{~km} / \mathrm{sec}$ in the construction of the ellipses. The intensity of the grey reflects the number of scattering ellipses that overlap. The envelope of the scatter- ing ellipses associated with the synthetic seismograms coincides with the previously determined blue reflector, whereas the envelope of the scattering ellipses associated with the data coincides with the red reflector.

These results can be further corroborated based on an independent measurement, the off-great-circle arrival angle $\psi$. With the help of the cartoon shown in Figure 7, this angle can be represented as

$$
\psi=\tan ^{-1}\left(\frac{2 H+L \sin \xi}{L \cos \xi}\right)-\xi .
$$




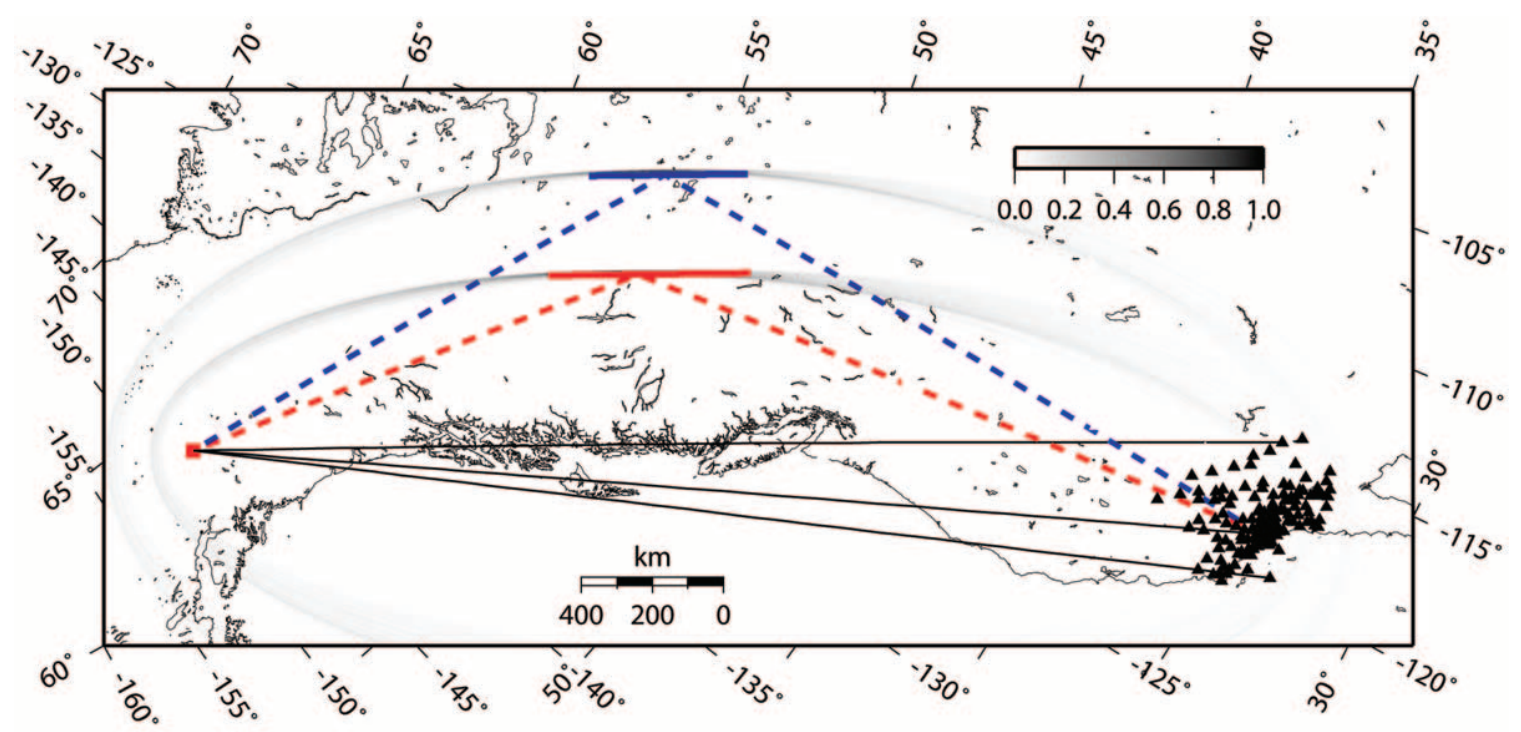

Figure 8. The travel time of the Rocky Mountain wave packet to a given station defines an ellipse (shown in gray tones) on which the scatterer responsible for the arrival must be located. The envelope of the scattering ellipses for all the stations delineates the reflector. The intensity of the gray reflects the number of scattering ellipses that overlap. The envelope of the scattering ellipses associated with the synthetic seismograms coincides with the previously determined blue reflector, whereas the envelope of the scattering ellipses associated with the data coincides with the red reflector (see also Fig. 7). Black triangles indicate the locations of the SCSN stations, and black lines denote typical great-circle paths.

For the data, the value predicted by equation (3) changes from $25^{\circ}$ to $28^{\circ}$ across the SCSN (Fig. 6, left panel). It matches the off-great-circle angles at stations with smaller source azimuths (ranging from $128^{\circ}$ to $134^{\circ}$ ) but is smaller for stations with larger source azimuths. The Rocky Mountain packet recorded by these stations has a larger off-greatcircle angle $\left(>30^{\circ}\right)$, suggesting that the associated reflection points are located south of the positions predicted based on the geometrical approach described previously. Because the predicted reflection points of these stations concentrate on the northern third of the blue bar, the analysis of the offgreat-circle angles suggests that the northern boundary of the reflection interface should be further south than that shown in Figures 2 and 7. Note that the Rocky Mountain packet is generated by all reflection points within its Fresnel zone, which gradually moves away from the northern boundary of the reflection interface as the source azimuth increases. As a result, the energy of the reflected wave is reduced. In the synthetic seismograms, we do observe that the peak amplitudes of the Rocky Mountain packet become smaller as the source azimuth increases, consistent with this prediction. Unfortunately, the synthetic seismograms do not constrain the southern end of the reflection interface, presumably because of the limited aperture of SCSN.

\section{Discussion and Conclusions}

Our simple analysis locates the reflector that is the origin of the synthetic "Rocky Mountain" packet at the eastern foot of the Rocky Mountains (Figs. 2, 7, and 8). For the synthetic seismograms, this result is consistent with the 20sec Rayleigh-wave phase-velocity map calculated based on crustal model CRUST2.0 and mantle model S20RTS (Fig. 7). The phase-velocity map features a linear lowvelocity zone along the high summits of the Rocky Mountains. Because the seismic waves originate on the west side of the mountains, the east side is an area of velocity increase and therefore a good candidate for the possible reflector. The highest part of the mountains is located south of $55^{\circ} \mathrm{N}$, which coincides roughly with the middle of the reflector that we attempted to locate. However, the data favor a lateral transition zone located $350 \mathrm{~km}$ further westward, where the high summits of the Rocky Mountains are located (Figs. 2, 7, and 8). Notice that the red bar is close to the Rocky Mountain Trench (Fig. 2), which is considered to be the western boundary of the Canadian Shield (Zhang et al., 1996). Therefore, our analysis of the observed data seems to suggest that the transition from the tectonic region to the Canadian Shield is much sharper than that included in the crustal and mantle models used in our simulations.

Comparisons between the data and 3D synthetic seismograms (Figs. 5 and 6) suggest that the ocean-continent transition in the CRUST2.0 model is too coarse to accurately model the propagation of Rayleigh waves at about $20 \mathrm{sec}$. For instance, at stations with an azimuth larger than $135^{\circ}$, the Oregon packet splits into three subpackets in the data, but retains a coherent shape in the synthetic seismograms (Figs. 2 and 7). The first subpacket in the data has a small 
amplitude and a large negative off-great-circle arrival angle and it arrives early (Fig. 6). This suggests that it may be a wave reflected by small-scale scatters west of the California coast. The remaining two subpackets have positive off-greatcircle arrival angles and arrive later. We speculate that they are waves reflected by the nearby Sierra Nevada Mountains (SNM) (Fig. 7).

As we have demonstrated in this study, the synthetic movies is a nice tool for looking at spatial and temporal correlations of seismic wave field, an essential feature for identifying anomalous wave packets. Note that the synthetic outputs used here are only a small part of what is actually produced by the 3D SEM simulations. They, in fact, can be used to investigate the wave motions at all depths and therefore to retrieve more precisely the reflection and refraction produced by the potential reflectors in the crust or mantle. Finally, the anomalous wave packets found in this study are apparently very sensitive to the crustal heterogeneities. It should be useful dataset to constrain the lateral velocity variations around mountains and plate boundaries in the future.

\section{Acknowledgments}

We thank Hiroo Kanamori for help in calculating the phase-velocity map and two anonymous reviewers for constructive suggestions. This research was supported in part by USGS Grant 04HQGR0048 and by the Gordon and Betty Moore Foundation. Seismic data were obtained from the Southern California Earthquake Data Center. The computations were performed at the Earth Simulator Center of JAMSTEC by Seiji Tsuboi. The figures were created with the Generic Mapping Tools (Wessel and Smith, 1998). This is Caltech Seismological Laboratory contribution 9090 and Caltech Tectonic Observatory contribution 4.

\section{References}

Bassin, C., G. Laske, and G. Masters (2001). The current limits of resolution for surface wave tomography in North America, EOS Trans. AGU 81, F897.

Capon, J. (1970). Analysis of Rayleigh-wave multipath propagation at Lasa, Bull. Seism. Soc. Am. 60, no. 5, 1701.

Cotte, N., H. A. Pedersen, M. Campillo, V. Farra, and Y. Cansi (2000). Off-great-circle propagation of intermediate-period surface waves observed on a dense array in the French Alps, Geophys. J. Int. 142, no. 3, 825-840.

Eberhart-Phillips, D., P. J. Haeussler, J. T. Freymueller, A. D. Frankel, C. M. Rubin, P. Craw, N. A. Ratchkovski, G. Anderson, G. A. Carver, A. J. Crone, T. E. Dawson, H. Fletcher, R. Hansen, E. L. Harp, R. A. Harris, D. P. Hill, S. Hreinsdottir, R. W. Jibson, L. M. Jones, R. Kayen, D. K. Keefer, C. F. Larsen, S. C. Moran, S. F. Personius, G. Plafker, B. Sherrod, K. Sieh, N. Sitar, and W. K. Wallace (2003). The 2002 Denali fault earthquake, Alaska: a large magnitude, slippartitioned event, Science 300, no. 5622, 1113-1118.

Evernden, J. F. (1953). Direction and approach of Rayleigh waves and related problems, part I, Bull. Seism. Soc. Am. 43, no. 4, 335-374.

Hauksson, E., P. Small, K. Hafner, R. Busby, R. Clayton, J. Goltz, T. Heaton, K. Hutton, H. Kanamori, J. Polet, D. Given, L. M. Jones, and D. V. Wald (2001). Southern California Seismic Network: Caltech/ USGS Element of TriNet 1997-2001, Seism. Res. Lett. 72, 697-711.

Ji, C., D. V. Helmberger, and D. J. Wald (2004). A teleseismic study of the 2002 Denali, Alaska, earthquake and implications for rapid strong motion estimation, Earthquake Spectra 20, no. 3, 617-663.
Knopoff, L., S. Mueller, and W. L. Pilant (1966). Structure of the crust and upper mantle in the Alps from the phase velocity of Rayleigh waves, Bull. Seism. Soc. Am. 56, 1009-1044.

Komatitsch, D., and J. Tromp (2002). Spectral-element simulations of global seismic wave propagation: II. 3-D models, oceans, rotation, and self-gravitation, Geophys. J. Int. 150, 303-318.

Laske, G. (1995). Global observation of off-great-circle propagation of long-period surface-waves, Geophys. J. Int. 123, no. 1, 245-259.

Lay, T., and T. C. Wallace (1995). Modern Global Seismology, Academic Press, New York.

Levshin, A., and K. A. Berteussen (1979). Anomalous propagation of surface waves in the Barents Sea at inferred from NORSAR records, Geophys. J. Int. 56, 97-118.

Nakanishi, I. (1992). Rayleigh-waves guided by sea-trench topography, Geophys. Res. Lett. 19, no. 24, 2385-2388.

Oliver, J. (1962). A summary of observed seismic surface wave dispersion, Bull. Seism. Soc. Am. 52, no. 1, 81-86.

Ritsema, J., H. J. van Heijst, and J. H. Woodhouse (1999). Complex shear wave velocity structure imaged beneath Africa and Iceland, Science 286, no. 5446, 1925-1928.

Snieder, R. (1986). 3-D linearized scattering of surface waves and a formalism for surface wave holography, Geophys. J. Int. 84, 581-605.

Tsuboi, S., D. Komatitsch, C. Ji, and J. Tromp (2003). Broadband modeling of the 2002 Denali fault earthquake on the Earth Simulator, Phys. Earth Planet. Interiors 139, no. 3-4, 305-312.

Wessel, P., and W. H. F. Smith (1998). New, improved version of Generic Mapping Tools released, Eos Trans. AGU 79, 579.

Woodhouse, J. H., and Y. K. Wong (1986). Amplitude, phase and path anomalies of mantle waves, Geophys. J. R. Astr. Soc. 87, no. 3, 753773.

Zhang, G. W., A. Hynes, and E. Irving (1996). Block rotations along the strike-slip Finlay-Ingenika fault, north-central British Columbia: implications for paleomagnetic and tectonic studies, Tectonics 15, no. 2 , 272-287.

Zhang, J. J., W. R. Walter, T. Lay, and R. S. Wu (2003). Time-domain pure-state polarization analysis of surface waves traversing California, Pure Appl. Geophys. 160, no. 8, 1447-1478.

\section{Appendix: Polarization Analysis}

Following the work of Cotte et al. (2000), we have developed a simple procedure to determine the polarization of waves recorded by a dense array. Before the analysis, vertical-component displacement seismograms recorded at SCSN stations were narrow bandpass filtered to reduce the effects of dispersion. In this study a Butterworth bandpass filter with corner frequencies of $0.04 \mathrm{~Hz}$ and $0.06 \mathrm{~Hz}$ is used. Next, for a given station A, we first look for neighboring stations whose distance to $\mathrm{A}$ is less than $R$. We then truncate the waveforms recorded at station $A$ using a time window with twice the length of the longest period of the data, and shift the record by the appropriate time to fit the waveforms at the selected neighboring stations. If we assume that between A and its neighbors the phase velocity $V$ is constant and that there is no significant variation in the incidence angle of the wave front $(\theta)$, then for a given neighboring station whose distance and azimuth to station $\mathrm{A}$ are $r$ and $\xi$, respectively, the expected time delay is

$$
d t=-\frac{r \cos (\theta-\xi)}{V}
$$


We perform a grid search for the optimal phase velocity $V$ and incidence angle $\theta$ so that the shifted waveforms best fit the records at all neighboring stations. We let the phase velocity change from $2 \mathrm{~km} / \mathrm{sec}$ to $4 \mathrm{~km} / \mathrm{sec}$ with an increment of $0.1 \mathrm{~km} / \mathrm{sec}$ and the incidence angle $\theta$ from $0^{\circ}$ to $359^{\circ}$ with an increment of $1^{\circ}$.

The distance $R$ used in this study is $70 \mathrm{~km}$. Because the phase velocity of 20-sec Rayleigh waves in Southern California is about $3.5 \mathrm{~km} / \mathrm{sec}$ (Fig. 7), this distance is about the wavelength of such waves. Because there are two unknowns, we need at least two neighboring stations to determine the result uniquely. This is the case for most SCSN stations except on the edges of the network. Let us also note that the method fails if $\mathrm{A}$ and all its neighboring stations fall approximately on a line, a situation that is very unlikely to happen in practice.
Division of Geological and Planetary Sciences

California Institute of Technology

Pasadena, California 91125

(J.T.)

Department of Earth Science

University of California, Santa Barbara

Santa Barbara, California 93106

(C.J.)

Institute for Research on Earth Evolution

Japan Agency for Marine-Earth Science and Technology

3173-25 Showa-machi

Kanazawa-ku Yokohama 236-0001, Japan (S.T.)

CNRS UMRS 212

Université de Pau et des Pays de l'Adour

Avenue de l'Université

64013 Pau Cedex, France

(D.K.)

Manuscript received 1 September 2004 